\title{
Near-Field Optical Flying Head with A Triangular Aperture
}

\author{
M. Hirata, M. Park, M. Oumi, K. Nakajima, and T. Ohkubo* \\ Research and Development Center, Seiko Instruments Inc., 563 Takatsuka-Shinden, Matsudo-shi, Chiba 270-2222, Japan \\ * School of Engineering, Toyo Univ., 2100 Kujirai, Kawagoe-shi, Saitama 350-8585, Japan
}

Heat assisted magnetic recording head needs near-field element as a micro heat generator and light guide structure. We have proposed a triangular aperture as a near-filed element. Computer simulation using finite difference time domain (FDTD) method shows that the triangular aperture has a near-field peak on the edge perpendicular to the polarization of the incident light. Therefore it achieves a near-field spot less than $20 \mathrm{~nm}$ (FWHM) in the direction of the polarization, which is not limited by the aperture size. SNOM and contact slider experiments in the previous study also show that the triangular aperture is effective. We have also proposed a compact light guide structure with an optical fiber. Using light guide structure with a polarization maintaining fiber and micro optics can achieve a thin and small $(1.6 \times 1.6 \times 0.7 \mathrm{~mm})$ near-field flying head. The head was fabricated. Signal readout was demonstrated in flying operation. It could read $300 \mathrm{~nm}$ L\&S patterns.

Key words: near-field, heat assisted magnetic recording, diffraction limit, FDTD, aspherical lens, polarization maintaining fiber

\section{Introduction}

Heat assisted magnetic recording (HAMR) has been proposed as a future recording technology to achieve a density over $1 \mathrm{~Tb} / \mathrm{inch}^{2}$. Extensive development of the head and the medium must be needed to realize this technology. We think that the key matters for HAMR head are the following three points.

(1) Near-field element as a micro heat generator

(2) Light guide structure

(3) Integrating near-field element and magnetic pole

Concerning (1), head for HAMR needs minute optical spot source as heat generator. Although an optical spot with a diameter less than $20 \mathrm{~nm}$ is necessary, it is impossible for conventional optics because of the diffraction limit. Therefore it is necessary to use near-field (NF) optics. When a light is introduced to a structure much smaller than the wavelength, electromagnetic field is clung onto the structure. This field is NF. Since the size of the NF is as same as the size of the structure regardless of wavelength of the light, very small optical spot can be generated beyond the diffraction limit.

Minute aperture has been used as a NF element, which is an optical aperture formed on a metallic film in small size less than the wavelength. However it has a disadvantage that NF generating efficiency is low. Especially, the smaller the size of the aperture becomes, the weaker NF intensity becomes drastically. To overcome this disadvantage, some structures have been proposed. For example, Matsumoto et al. proposed nanobeak structure, and showed that it will generate a $\mathrm{NF}$ spot less than $20 \mathrm{~nm}$ in diameter in $20 \%$ efficiency by FDTD (finite domain time difference) simulation ${ }^{1)}$.

Concerning (2), the head also needs a light guide structure from a light source to a NF element. In previous study, a laser and lenses were arranged above the flying head discretely, because they were too large to build in. We think that the head should have a thin and small light guide structure that does not affect the flying stability with low flying height, and has high efficiency. For example, Gage et al. proposed grating coupler which receives the light from a laser and planner SIM (solid immersion mirror) to concentrate the light ${ }^{2}$.

Concerning (3), distance between magnetic field and optical spot is important for HAMR. They must be very close to each other. Although several researches of optimum layout of them have been reported ${ }^{3)}$, research of integrated structure of magnetic pole and optical spot is scarce yet. For example of the integrated structure, Miyanishi et al. proposed SMASH (surface plasmon and magnetic field applicable synchronously hybridized) head ${ }^{4)}$ that has curved metallic wire and ledge structure. The wire generates magnetic field. NF is localized on the ledge structure. They demonstrated HAMR writing on $\mathrm{TbFeCo}$ medium.

We have focused on the key (1) and (2) so far. We have already proposed a triangular aperture as near-field element, which leads to (1) and a light guide structure using horizontally placed optical fiber, which leads to (2). In this paper firstly we studied the characteristics of the triangular aperture with FDTD simulation $^{566}$. Secondly, we demonstrated signal readout test with flying head that has these two features. The key (3) will be a future work.

\section{Triangular aperture}

Triangular aperture has a NF peak on the edge perpendicular to the polarization of the incident light. It can realize high resolution and $\mathrm{S} / \mathrm{N}$ ratio beyond the limit of an aperture size. The aperture is formed on top of tetrahedral protrusion with quartz $\left(\mathrm{SiO}_{2}\right)$ core and aluminum shading film. The shading film on the apex is 
removed, and the apex of the core is exposed. The thickness of the shading film is $300 \mathrm{~nm}$. Figure 1 shows computer simulation of $\mathrm{NF}$ distribution $10 \mathrm{~nm}$ above the triangular aperture (size 150nm) when a linearly polarized light is introduced to the aperture. The light of $488 \mathrm{~nm}$ wavelength is introduced from the bottom face of the quartz core, and $\mathrm{NF}$ is distributed from the aperture to the air. A single peak on the edge perpendicular to the polarization direction is shown, and is narrow along the polarization direction ( $\mathrm{X}$ direction), and as wide as the length of the edge in $\mathrm{Y}$ direction.

Figure 2 shows the NF spot profile in $\mathrm{X}$ direction. The line (a) indicates the result shown above. The peak indicates the localized NF. The spot size in full width at half maximum is $16 \mathrm{~nm}$. On the contrary, the line (b), when the polarization of the incident light is parallel to the edge, the NF is not localized. The spot size is 110 $\mathrm{nm}$ at this condition, which is almost equivalent to the aperture size. The result shows that the triangular aperture achieves a high resolution beyond the aperture size by polarization control.

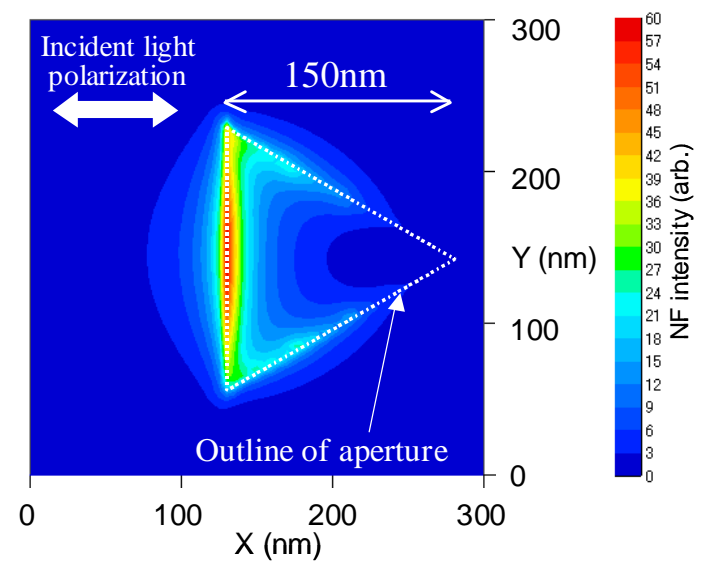

Fig. $1 \mathrm{NF}$ distribution at $10 \mathrm{~nm}$ above the aperture

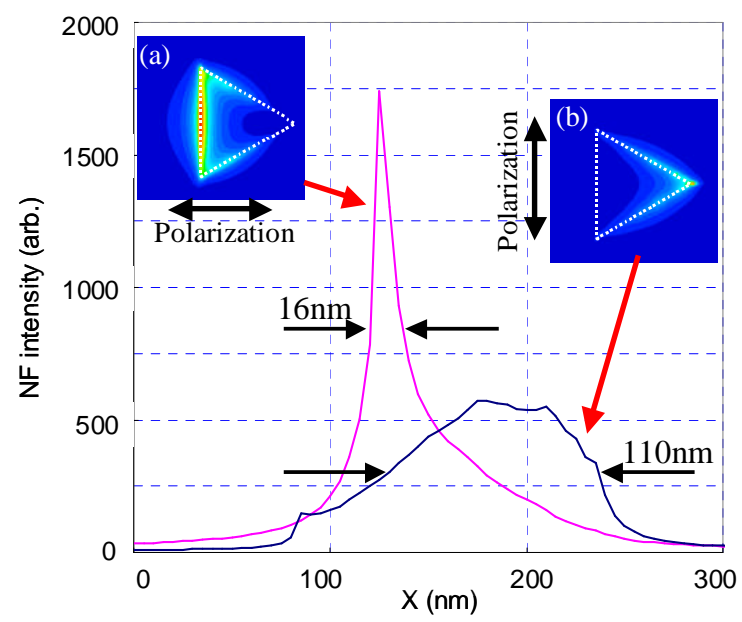

Fig. 2 NF profile at $10 \mathrm{~nm}$ above the aperture

The localized NF decays quickly as distance from the aperture is increased. Figure 3 shows dependence of
NF spot size (FWHM) on distance from the aperture. The spot size increases with the distance. When the distance is $10 \mathrm{~nm}$, the spot size is less than $20 \mathrm{~nm}$. On the contrary, when the distance is $40 \mathrm{~nm}$, the spot size is about $100 \mathrm{~nm}$. The result suggests that aperture-tomedium spacing must be reduced for high resolution.

Triangular aperture has another advantage that it does not need ultra-fine fabrication technique. We have already demonstrated these advantages with SNOM (scanning near-field optical microscope) probes and contact heads, and these experiments showed the triangular aperture is effective ${ }^{7)}$.

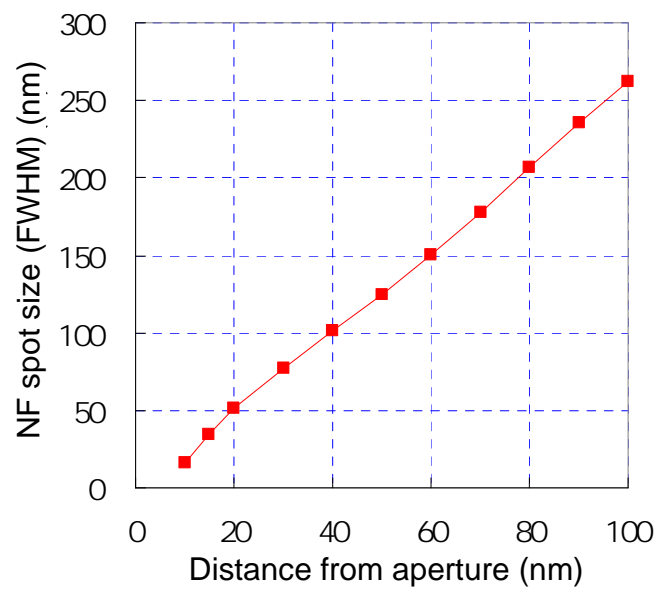

Fig.3 NF spot size versus distance from the aperture

\section{Light guide structure}

Our NF optical flying head has compact light guide structure using a horizontally placed optical fiber and micro optics ${ }^{8}$. This structure enables NF head with optics to be installed in HDD. Figure 4(a) shows the structure of our NF head, whose dimension is $1.6 \times 1.6 \times 0.7 \mathrm{~mm}$. The head consists of a slider, a mirror substrate, a polarization maintaining (PM) fiber and a suspension. The slider made of quartz has a micro lens on the upper side. It has the triangular aperture and air bearing surface (ABS) on the bottom side. The mirror substrate has a micro mirror and a V-groove. These are formed by anisotropic etching of the $\mathrm{Si}$ substrate. The micro mirror consists of etched $\mathrm{Si}$ (111) plane and $\mathrm{Al}$ film. The PM fiber is fixed on the V-groove. The light from the fiber is reflected toward the aperture by the mirror and condensed by the micro lens and introduced to the aperture.

Figure 4(c) shows the optical design of this structure. The mirror is not shown in this figure. NA of the PM fiber is 0.11. Distance from the PM fiber to the aspherical lens is $415 \mu \mathrm{m}$. Distance from the lens to the aperture is $300 \mu \mathrm{m}$. NA of the lens is 0.22 . The microlens is aspherical whose effective diameter is $100 \mu \mathrm{m}$. It is formed on quartz substrate by gray scale etching technique. Focusing efficiency within $\phi 3 \mu \mathrm{m}$ is $55 \sim 80 \%$.

The PM fiber has a couple of stress members to 
maintain polarization. The core of the fiber is in between the stress members. The line connecting the stress members and the core is defined as slow-axis, the line perpendicular to the slow-axis is defined as fast-axis. Polarization is maintained along slow and fast axis. The slow-axis of the PM fiber is perpendicular to the ABS. Polarization parallel to the slow-axis is maintained in the PM fiber, at the micro mirror and the micro lens. Therefore, NF is localized on the edge of the aperture, and resolution becomes high.

The ABS is step-flat positive-pressure type that has a couple of rails parallel to the suspension. Flying height of this type of flying head with an optical fiber was measured using an acoustic emission (AE) sensor ${ }^{9}$. Minimum flying height at trailing edge was estimated to be $30 \mathrm{~nm}$. The aperture is protruded from the ABS by $20 \mathrm{~nm}$ so that effective spacing between the aperture and the disk is decreased. Considering the inclined angle of flying attitude, the spacing between the aperture and the medium is $15 \mathrm{~nm}$ at the minimum.

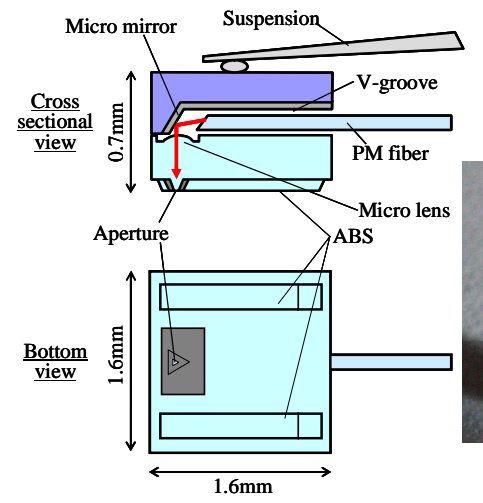

(a) Flying head with light guide structure

(b) Photograph of flying head
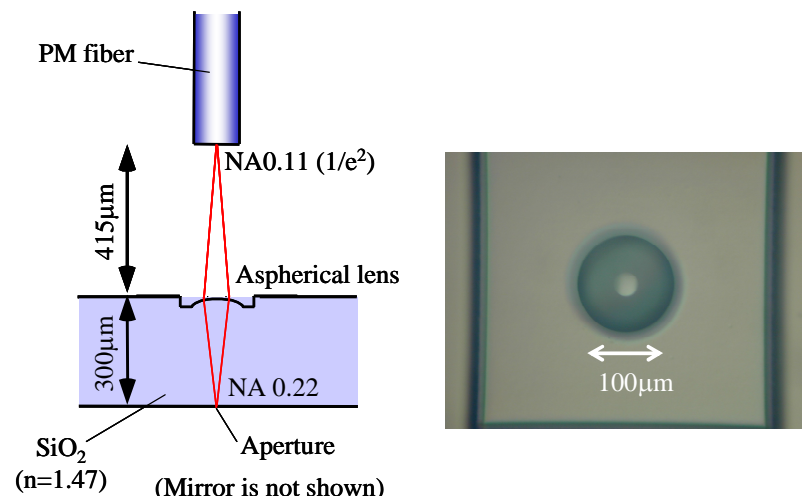

(c) Optical design

(d) Aspherical lens

Fig.4 Flying head with light guide structure

\section{Signal readout test}

The NF flying head with the triangular aperture and the light guide structure was fabricated as shown in Fig. 4(b). Signal readout test was demonstrated using the head and a mock medium with chromium lines and spaces (L\&S) patterns. Fig.5(a) is schematics of the setup, and (b) is the photograph. The mock medium is attached to a spindle motor and is rotated. The head is approached to the bottom surface, which has L\&S patterns, of the medium, and flies above the medium. The light from $\mathrm{Ar}^{+}$laser, whose wavelength is $488 \mathrm{~nm}$, is introduced to the head through a PM fiber. The NF from the triangular aperture is scattered by the patterns on the medium and detected by a photomultiplier through a microscope.

Figure 5(c) is an SEM image of the triangular aperture that is used for this experiment. The size in the scan direction is $120 \mathrm{~nm}$. Far field efficiency is $3.5 \times 10^{-5}$. Although near field efficiency was not measured, it should be much bigger than this.

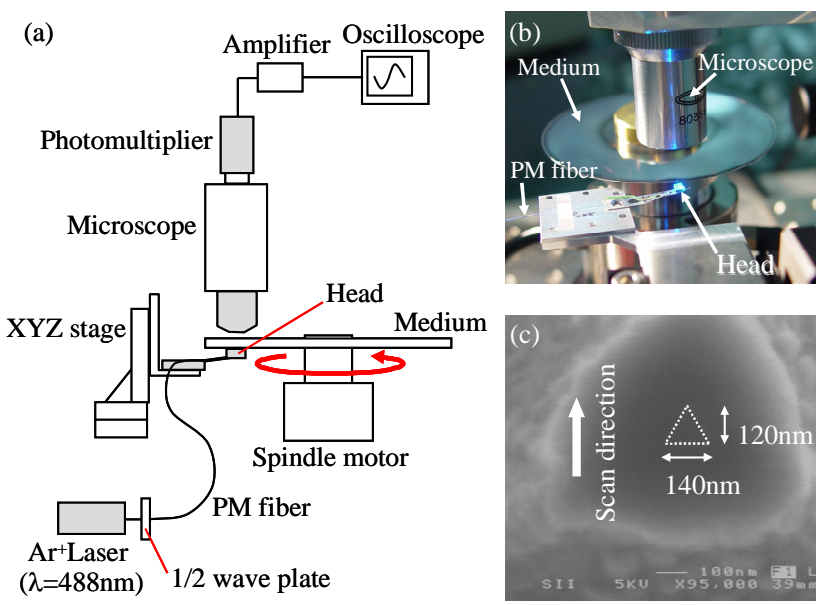

Fig. 5 Setup for signal readout test (a) Schematics (b)Photograph (c)SEM image of triangular aperture

Figure 6 shows the structure of the mock medium. The medium consists of a $\phi 2.5$ inch transparent glass disk, a 30-nm-thick chromium layer, a 10-nm-thick carbon protective layer, and a 1-nm-thick lubricant layer. Patterns are formed on the chromium layer. The patterns consist of 100, 150, 200, 300-nm-wide lines and spaces (L\&S). Each L\&S pattern has 20 periods. The patterns are formed by electron beam lithography. The triangular aperture scans these patterns from left to right in this figure. Linear velocity of the medium is $2.20 \mathrm{~m} / \mathrm{s}$. The aperture to medium spacing is estimated to be $30 \mathrm{~nm}$.

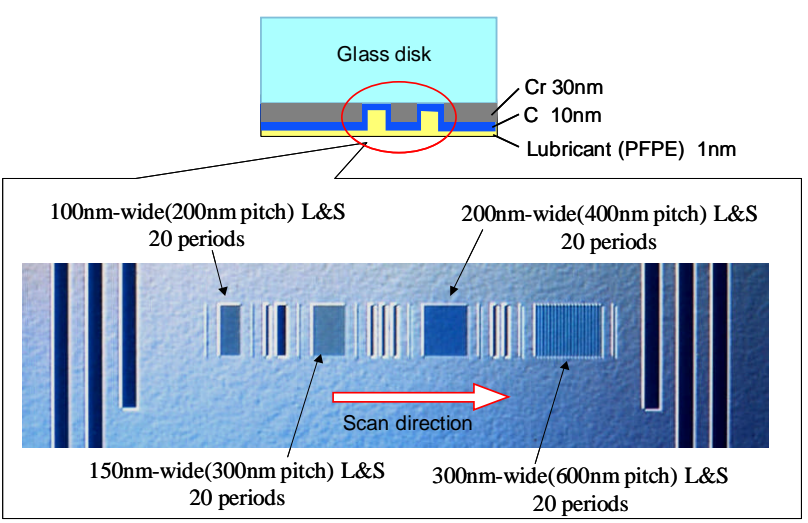

Fig. 6 Structure of mock medium 
Figure 7(a) is the signal from $300 \mathrm{~nm}$ L\&S. When a scattered light is detected, voltage of the signal becomes low. The 20 period signals correspond to 20 period patterns are detected. This result shows that the head with the triangular aperture and the light guide structure can detect signal in flying operation, and it can read $300 \mathrm{~nm}$ patterns.

Figure $7(\mathrm{~b})$ is the signal from $150 \mathrm{~nm} \mathrm{L \& S}$. It seemingly can read these patterns, but the signal has only 19 periods from 20 period patterns. It shows that the scattered light from adjacent two patterns is detected at the same time. Therefore, the resolution of the head is estimated to be worse than $300 \mathrm{~nm}$.

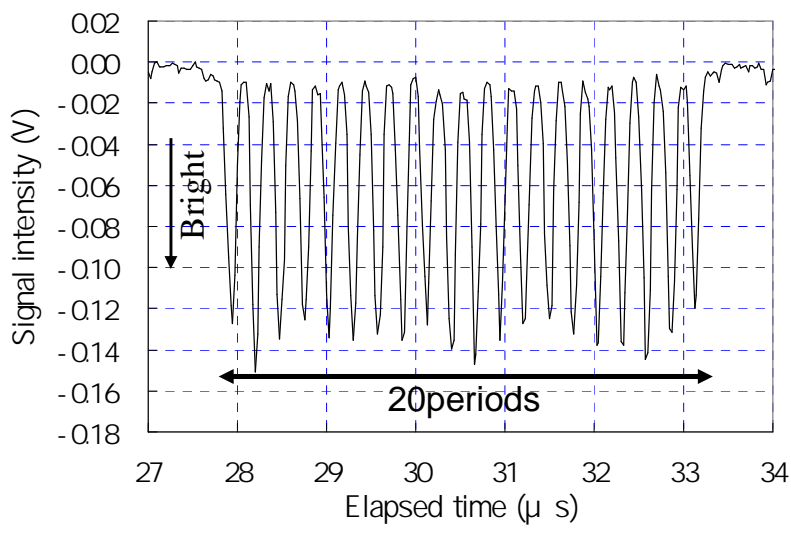

(a) Signal from $300 \mathrm{~nm}$ L\&S

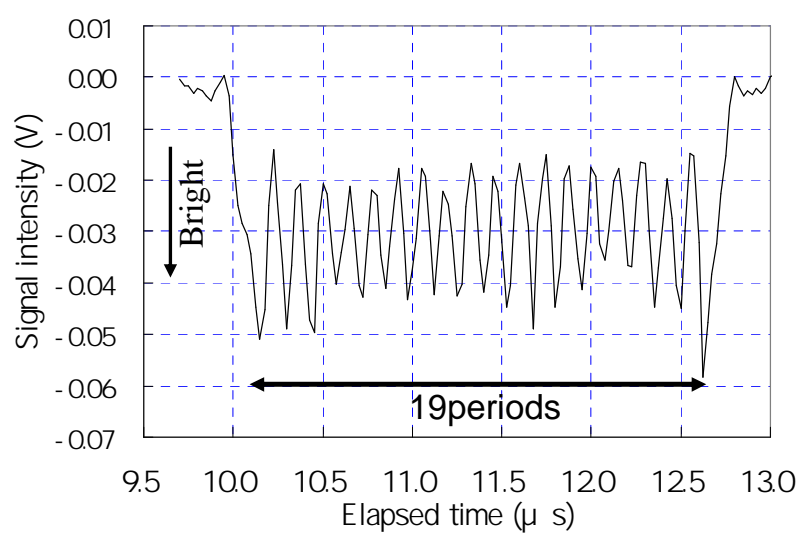

(b) Signal from $150 \mathrm{~nm}$ L\&S

Fig. 7 Readout signal

This result suggests that the spot size on the medium is more than $300 \mathrm{~nm}$. As has been mentioned, aperture to medium spacing is estimated to be $30 \mathrm{~nm}$ in this experiment. When the distance from the aperture is $30 \mathrm{~nm}$, the spot size is less than $100 \mathrm{~nm}$ according to the simulation in Fig.3.

The effective spacing of this experiment is the distance between the edge of the aperture and the $\mathrm{Cr}$ patterns, and is more than $30 \mathrm{~nm}$, because the top of the tetrahedral tip and the edge of the aperture has altitude difference. NF is localized on the edge, and the medium has carbon overcoat. Considering these reasons, the effective spacing might be about $100 \mathrm{~nm}$. If that is the case, the simulation in Fig. 3 says that the spot size is almost $300 \mathrm{~nm}$, so that the resolution I have mentioned is not improbable. In future work, we must care about not only flying height of the slider but also these points.

\section{Conclusion}

HAMR head needs NF element as a micro heat generator and light guide structure. We have proposed a triangular aperture and a compact light guide structure with a PM fiber. In this paper, computer simulation shows that the triangular aperture has a NF peak on the edge perpendicular to the polarization of the incident light. Therefore it achieves less than $20 \mathrm{~nm}$ spot size (FWHM) in the direction of the polarization, which is not limited by the aperture size. SNOM and contact slider experiments in the previous study also show that the triangular aperture is effective.

Using light guide structure with optical fiber and micro optics can achieve a thin and small (1.6x1.6x 0.7 $\mathrm{mm}$ ) NF flying head. The head was fabricated. Signal readout was demonstrated in flying operation. It could read $300 \mathrm{~nm}$ L\&S patterns.

HAMR head also needs integrating near-field element and magnetic pole. It will be a future work.

Acknowledgements A part of this paper belongs to "Terabyte optical storage technology" project that OITDA contracted with The Ministry of Economy Trade and Industry of Japan (METI) in 2002 and contracted with The New Energy and Industrial Technology Development Organization (NEDO) since 2003 based on funds provided from METI.

\section{References}

1) T. Matsumoto, Y. Anzai, T. Shintani, K. Nakamura, and T. Nishida: Opt. Lett., 31, 2, 259 (2006).

2) E. C. Gage, C. Peng, T. Rausch, W. Challener, B. Mihalcea, M. Seigler, K. Pelhos, and T. McDaniel : MORIS 2006 Workshop, (2006).

3) F. Akagi, M. Igarashi, K. Ishikawa, A. Nakamura, M. Mochizuki, H. Saga, and T. Matsumoto: Tech. Rep. IEICE, MR2003-23 (2003) [in Japanese].

4) S. Miyanishi, N. Iketani, K. Takayama, K. Innami, I. Suzuki, T. Kitazawa, Y. Ogimoto, Y. Murakami, K. Kojima, A. takahashi: IEEE Trans. Magn., 41, 10, 2817 (2005).

5) K. S. Yee: IEEE Trans. Antennas Propag., 14, 302 (1966).

6) M. Oumi: Opt. Rev., 8, 54 (2001).

7) M. Hirata, M. Oumi, K. Shibata, K. Nakajima, and T. Ohkubo: IEICE Trans. Electron., E90-C, 1, 102 (2007).

8) M. Hirata, M. Oumi, K. Nakajima, and T. Ohkubo: Jpn. J. Appl. Phys., 44, 3519 (2005).

9) T. Ohkubo, M. Hirata, M. Oumi, K. Nakajima, and T. Hirota: Proc. SPIE, 6050, 6050C (2005).

Received Oct. 11, 2007; Revised Dec. 04, 2007;

Accepted Dec. 18, 2007 